\title{
SoTL and the Institutional Review Board: Considerations before navigating the application process for classroom research in higher education
}

\begin{abstract}
Kathryn E. Linder ${ }^{1}$, E. Deborah Elek ${ }^{2}$, and Lucia Calderon ${ }^{3}$
Abstract: One of the more challenging areas of Scholarship of Teaching and Learning (SoTL) research can be navigating the components of human subjects research protections implemented by the Institutional Review Board (IRB). The authors of this article, a faculty developer and a current and former research compliance coordinator, discuss the history of IRB in relation to SoTL research and explicate some of the foundational components of IRB protocols for SoTL projects. In particular, the authors explore what constitutes "research" for SoTL projects, explain the different IRB types of review, and offer some sample SoTL projects with respect to their IRB implications.
\end{abstract}

Keywords: Institutional Review Board, Scholarship of Teaching and Learning

\section{Introduction}

One of the more challenging areas of Scholarship of Teaching and Learning (SoTL) research can be navigating the components of human subjects research protections established by the Office of Human Research Protections and implemented by the Institutional Review Board (IRB). The literature has shown that interacting with the IRB can be a challenge for faculty who are unfamiliar with human subjects research protections and/or the scholarship of teaching and learning (see, for example, Lopus, Grimes, Becker, \& Pearson, 2007; Wright, Finelli, Meizlish, $\&$ Bergom, 2011). Despite the pervasiveness of SoTL in higher education (evidenced through conferences such as IS-SoTL, publications such as the Journal on Excellence in College Teaching and the Journal of the Scholarship of Teaching and Learning, and the growth of SoTL initiatives at research institutions such as the University of Michigan's Investigating Student Learning Grant program), guidelines for faculty regarding how to navigate the human subjects research requirements for SoTL projects. For example, McKinney (2007) provides an excellent and extensive guide for designing SoTL research projects, but only mentions IRB in passing as one of many challenges "on the horizon for starting (and completing!) SoTL projects" (p. 25). As we explored the SoTL literature, we found only one article (Pritchard, 2001) that included guidelines aimed at SoTL researchers that both discussed the components of IRB review while also providing examples of how this might apply in a SoTL context. We found no guidelines that were written from the combined perspectives of IRB professionals and faculty developers.

In the remainder of this article, we use our shared experience to discuss the history of IRB in relation to SoTL research and explicate some of the foundational components of IRB review for SoTL projects. In particular, we explore what constitutes "research" as it relates to SoTL projects, explain the different IRB types of review, and offer some sample SoTL projects

\footnotetext{
${ }^{1}$ Director of the Center for Teaching Excellence, Suffolk University, klinder@suffolk.edu

${ }^{2}$ Research Compliance Manager, Suffolk University, edelek@suffolk.edu

${ }^{3}$ Medical Affairs Manager, EMD Serono, Inc., luciaverdaguer@hotmail.com
} 
with their IRB implications. We hope that this reflective piece will offer some clarification for faculty members, particularly those who are new to SoTL research or who are first-time IRB applicants.

\section{SoTL as Human Subjects Research}

As Hutchings argues, the ethical issues embedded within SoTL research "are not simply occasions for caution, but windows into our aspirations and values as educators" (Hutchings, 2003, p. 28). SoTL researchers are being asked to engage in questions of ethics and values within their teaching and research (Pritchard, 2002). The possible risk to students as a potential vulnerable population must be weighed against the need to assess the effectiveness of teaching and learning. Swenson and McCarthy (2012) elaborate, "faculty must attend to competence in teaching by using scientifically sound practice and safeguarding the very students who are participants in the pedagogical research" (p. 22). Pecorino, Kincaid, \& Gironda (2008) call for a separate "ethical review process" for classroom research (p. 2). While students can certainly be empowered by participating in research on teaching and learning, particularly when they participate as co-PIs or co-authors, students have also been perceived as a vulnerable population in three main areas: the power differential between students and teachers who will be grading current students who are also research subjects (Hammack, 1997; Pecorino, Kincaid, \& Gironda, 2008); the value judgments made by teachers who incorporate new texts, assignments, assessments, technologies, or learning procedures for the purpose of SoTL research (Burman \& Kleinsasser, 2004; Pecorino et al., 2008); and the "dual-role conflict" (Hammack, 1997) created when "the research may be intended, in part, to further the career of the teacher/researcher by generating publications or conference presentations" (Pecorino et al., 2008, p. 5; see also Burman \& Kleinsasser, 2004). Submitting research on classroom practice to the IRB for review is a crucial step for investigators who wish to ensure that their students are protected as research subjects. Moreover, many SoTL journals require that researchers have obtained IRB approval before they will publish research based on data collected from human subjects.

The Scholarship of Teaching and Learning (SoTL) is a body of research and literature that has grown rapidly in the decades after its initial definition. At present, several definitions of SoTL exist. Even as McKinney (2007) notes the importance of "recogniz[ing] the diversity in definitions or understandings of SoTL that exist even among experts in the field" (p. 5), some common features have emerged across a variety of SoTL definitions. Based on these common features, we define SoTL research as the following:

- An inquiry or investigation of classroom practice [including online environments] in higher education (Huber and Hutchings, 2005; Menges \& Weimer, 1996),

- Using a systematic and intentional (and perhaps disciplinary) methodology (Cambridge, 1999; Kreber, 2001),

- Resulting in a scholarly product, such as a conference presentation or journal article manuscript, to be publicly disseminated (Huber \& Hutchings, 2005; Cambridge, 1999; Kreber, 2001; Richlin, 2001; Savory, Burnett \& Goodburn, 2007; Shulman, 1999),

- That can be built upon by colleagues also engaging in SoTL research (Shulman, 1999).

As a faculty developer and a current and former research compliance coordinator who often partner with one another to help faculty navigate the relationship between SoTL and the requirements necessary for conducting human subject research, we have found that a shared definition between our offices has been a helpful component of this work. After working 
together over several years on shared projects, we looked to the literature for a "how-to" guide that we could offer to faculty who were novices in the field of SoTL research. With the exception of Pritchard (2001, 2002), we struggled to find this kind of resource.

The literature reports that IRB approval can be perceived as a barrier for instructors wishing to contribute to the scholarship of teaching and learning (Lopus et al., 2007; Wright et al., 2011) with some arguing that "the cost of the [research ethics committee] process for researchers conducting classroom-based studies may outweigh the benefits to the student subjects and other interested parties" (Lopus et al., 2007 p. 69). While the IRB as a perceived barrier is not unique to SoTL researchers (researchers in other fields may experience similar confusion regarding the application process), it is important to note that researchers who are new to SoTL may be experiencing the IRB for the first time. Since SoTL research occurs across a wide variety of disciplines, researchers applying for IRB approval may not have received any previous training or have any past experience of working with the IRB. Moreover, the IRB application, protocol, and review process can be confusing to navigate because the IRB's regulatory definition of "research" can differ from common definitions of research used by faculty members (Pritchard, 2002; see Table 1).

Table 1

Comparative Definitions of Research

\begin{tabular}{|c|c|c|}
\hline $\begin{array}{l}\text { Faculty Perspective(s) of } \\
\text { Research }\end{array}$ & IRB Perspective of Research & $\begin{array}{lll}\text { SoTL } & \text { Perspective } & \text { of } \\
\text { Research } & & \\
\end{array}$ \\
\hline $\begin{array}{l}\text { "Systematic self-critical } \\
\text { inquiry" that is "made public" } \\
\text { (Stenhouse, 1981, 103; 104) } \\
\text { "Gather[ing] information to } \\
\text { answer a question that solves } \\
\text { a problem" (Booth, Colomb, } \\
\text { \& Williams, 2008, p. 10) } \\
\text { "Contributes not only to the } \\
\text { stock of human knowledge } \\
\text { but also to the intellectual } \\
\text { climate of the university. Not } \\
\text { just the outcomes, but the } \\
\text { process, and especially the } \\
\text { passion, give meaning to the } \\
\text { effort" (Boyer, 1990, p. 17) }\end{array}$ & 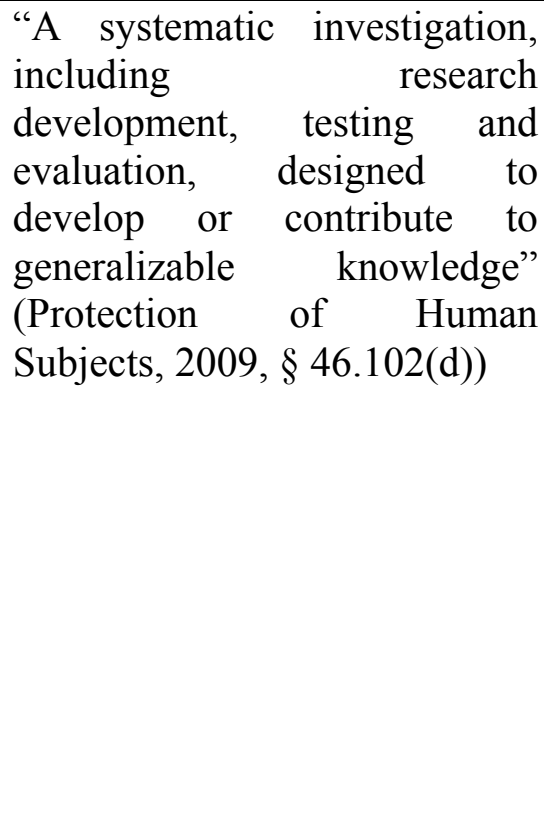 & $\begin{array}{l}\text { An inquiry or investigation of } \\
\text { classroom practice in higher } \\
\text { education, using a systematic } \\
\text { and intentional (and perhaps } \\
\text { disciplinary) methodology, } \\
\text { resulting in a scholarly } \\
\text { product to be publicly } \\
\text { disseminated that can be built } \\
\text { upon by colleagues also } \\
\text { engaging in SoTL research. }\end{array}$ \\
\hline
\end{tabular}

The first step toward a successful relationship between SoTL researchers and IRBs is to have a common understanding of the regulatory framework in which IRB professionals are trained to think about research. Research with human subjects has been regulated by the federal government since the 1970s with the passage of the National Research Act of 1974. The act was passed in response to public outcry amidst the 1973 Congressional hearings on human 
experimentation, an effort led by the late Senator Edward Kennedy. These hearings put in the forefront several instances of research conducted without any ethical standards in place to protect human participants. Some of the most cited examples are the Tuskegee syphilis study (Schuman, Olansky, Rivers, Smith, Rambo, 1955), the Tearoom trade study (Humphreys, 1970), and the Milgram studies of obedience (Milgram, 1963). The 1974 Act established the National Commission for the Protection of Human Subjects of Biomedical and Behavioral Research, which in 1978 issued the Belmont Report. This report laid out the three basic ethical principles for the conduct of human subjects research: respect for persons, justice, and beneficence. In 1984, with this report as the foundational background, the federal agencies responsible for the oversight of human subjects research promulgated the "Common Rule."

The Common Rule is the federal policy for the protection of human subjects involved in research and what all IRB professionals use as their regulatory framework. The Common Rule has been adopted by several federal agencies and it dictates the requirements for assuring compliance by research institutions as well as the requirements for obtaining and documenting informed consent of research participants. The Common Rule also regulates how IRBs are created, how they function, and the criteria used for IRBs to review and approve human subjects research. The key terms here are "research" and "human subjects." Historically, as the National Commission title suggests, these terms have been understood to apply solely to biomedical and social/behavioral research. However, as more higher education institutions formalized IRB functions in a centralized office, an increased concern about conducting ethical research meant an expanded interpretation of the regulatory meaning of research. Under this expanded interpretation, a SoTL project that otherwise does not fall under traditional psychological or sociological human behavioral research may still necessitate an IRB inquiry. SoTL researchers should also note that their disciplines might have additional ethics codes that may apply to their classroom research (see, for example, Swenson \& McCarthy, 2012).

\section{What Constitutes "Research" and "Human Subjects" according to the federal regulations?}

In The Nature of Research, Brew argues "there is no one thing, nor even a set of things, which research is. It is obviously a complex phenomenon" (Brew, 2001a, p. 21). Brew's own scholarship, in which she explores the relationship between research and teaching, has attempted to further clarify how academics experience research in a variety of ways from different perspectives. For example, based on one study of 57 researchers, Brew identifies four qualitative conceptions of research that she refers to as "domino," "layer," "trading," and "journey" (Brew, 2001 b, p. 280). As varying definitions of research are a common foundation for academic work, particularly across diverse disciplines, faculty may feel startled when the validity of their scholarship as research is questioned by an IRB professional.

The IRB defines research as "a systematic investigation, including research development, testing and evaluation, designed to develop or contribute to generalizable knowledge" (Protection of Human Subjects, 2009, § 46.102(d)). Each part of this definition is an important component that influences the determination of whether a SoTL project falls within the purview of the IRB. An IRB professional, when reviewing a SoTL project, should first ask:

1) Does this meet the regulatory definition of research?

In order to answer this question, an IRB professional will apply all parts of the definition of research to the project with a specific focus on the key phrases "systematic" and "designed to 
develop or contribute to generalizable knowledge." Another question that may be asked is "What is the intended purpose of the investigation?"

Example: If while teaching an introduction to biology course, an instructor wants to see if her students learn better with new online laboratory modules than from a traditional textbook, this most likely will not meet the regulatory definition of research. While this might represent a systematic investigation because she plans to use a scientific method to collect and analyze data between two semesters of classes that use the two different instructional methods, it is equally important to note that the intention behind the analysis is solely to improve her own teaching practices and her students' learning experience. As opposed to being designed to contribute to general knowledge about best teaching practices for biology courses, this inquiry is too limited in scope to meet the regulatory definition of research. When looking at SoTL projects in relation to the regulatory definition of research, one of the most important things to remember is that it must meet the definition in its entirety.

Using this example, with the same parameters described above for the inquiry, rather than solely trying to improve her teaching, the instructor now intends to design the study so that her results will be appropriate for publication and contribute to generalizable knowledge regarding the benefits of using technology to help students learn. Because her study design now includes the intention to contribute to this body of knowledge that can be used by other biology teachers at other institutions and in diverse classroom contexts, it now is more likely to meet the regulatory definition of research. However, it is important to note that designing a study with the intention to publish the results does not automatically make the project generalizable. For instance, if the design of a classroom research study is so unique that the knowledge it creates cannot be transferred to other classroom contexts, the intention to publish will have no impact on the determination of whether the project falls within the regulatory definition of research. One of the most crucial differences between faculty understandings of research and the regulatory definition (and perhaps one of the most frustrating differences), is that publication equates research for faculty, but this is not always the case for the regulatory definition. For example, non-academic publications (for example, articles in newspapers) describing an instructor's experience with their students in a non-identifiable way would not be classified as "research" that would need to be approved by the IRB.

Upon determining that a SoTL project meets the definition of research, an IRB professional will next ask:

(2) Does this meet the definition of human subjects?

"Human Subjects" are defined as "a living individual about whom an investigator (whether professional or student) conducting research obtains (1) data through intervention or interaction with the individual, or (2) identifiable private information" (Protection of Human Subjects, 2009, $\S 46.102(\mathrm{f})$ ).

If the answer to both of these questions is no, then the project does not require further inquiry by the IRB. However, if the answer to either question is yes, then the definition of human subjects has been met.

Broken down even further, the first part of the definition of human subjects references obtaining data through "intervention or interaction." The federal definition explains this component as "both physical procedures by which data are gathered (for example, venipuncture) and manipulations of the subject or the subject's environment that are performed for research purposes" as well as "communication or interpersonal contact between investigator and subject" (Protection of Human Subjects, 2009, § 46.102(f)). In addition to face-to-face contact between 
researcher and subject, data collection through online venues or non-face-to-face procedures also meets the regulatory definition of human subjects. Examples of this kind of data collection might include online surveys, phone interviews, and surveys collected via email or postal mail.

SoTL projects often meet the definition of human subjects because they involve interventions or interactions with students such as manipulating the classroom environment.

Example: An instructor wants to learn about the differences in his students' classroom experience when they are exposed to fresh air versus not exposed. He designs a SoTL project to measure the differences using a classroom that in one semester will have opened windows and in the following semester, the windows will be closed. Assuming this project has already met the regulatory definition of research, it would also meet the definition of human subjects because the classroom environment has been manipulated for research purposes.

Another important component of the definition of human subjects is the reference to "identifiable private information." Private information is further explained in the federal regulations as "information about behavior that occurs in a context in which an individual can reasonably expect that no observation or recording is taking place, and information which has been provided for specific purposes by an individual and which the individual can reasonably expect will not be made public" (Protection of Human Subjects, 2009, § 46.102(f)). Private information must also be classified as "individually identifiable (i.e., the identity of the subject is or may readily be ascertained by the investigator or associated with the information)" (Protection of Human Subjects, 2009, § 46.102(f)). If a SoTL project does not obtain data through intervention or interaction, but does include the use of identifiable private information, then it would meet the definition of human subjects.

Example: A Sociology professor has given her students a journal assignment in which they are supposed to record their observations during their commutes to and from campus and connect what they observe to what they are learning in class. This journal assignment is explained by the professor to be a confidential communication between the instructor and her students. The class in which the assignment is originally used has six students. After the third iteration of the course, and the third cycle of using this assignment with additional groups of 20 and 25 students, respectively, the professor begins to notice a trend in her students' journal entries regarding their observations of everyday violence and she would like to design an exploration of this pattern. Assuming that her investigation meets the regulatory definition of research, there are several questions related to the human subjects definition that an IRB professional might consider.

1) Were the journals collected through interaction or intervention with the students for the purpose of research?

No. The data was collected as a classroom assignment and not for research purposes.

2a) Are the journals private information?

Yes. The students were fulfilling a specific writing assignment, which they reasonably expected would be kept private by their instructor because the professor described the journals as confidential.

2b) Are the journals identifiable?

Maybe. The first group of journal entries were submitted by a small group of students. Depending on the level of detail given in the journal entries, the data may be identifiable. This is less likely, but still possible, in the larger groups of students. 
3) Does this investigation meet the regulatory definition of human subjects?

Maybe. The human subjects definition has two parts, interaction or intervention and identifiable private information; at least one of these criteria must be met. If the answer to question $2 b$ is no, then neither part has been met. However, if the answer is yes, then this investigation meets the definition of human subjects.

Based on the above examples, it is clear that applying the regulatory definitions of research and human subjects is not always a simple process. This is why we recommend always establishing a relationship with your institution's IRB professional or research compliance office and consulting with them ahead of time when there is uncertainty about whether IRB review is needed. Below, we elaborate on the different types of IRB oversight.

\section{Exempt vs. Expedited vs. Full IRB Review of Research}

If it is determined that your classroom research meets both the regulatory definitions of research and human subjects, you will want to continue with an application for IRB review. In this section, we will further define and explain the different criteria and thresholds that may suggest the appropriate type of IRB review. There are three main types of review that can be applied to studies involving human subjects: exempt, expedited, and full review. Each type of review can involve different paperwork and requirements for the researcher based on local institutional policies. Below, we define each of these types and offer examples of classroom research projects that meet criteria for a particular type of review. It should be noted that while regulations provide minimum criteria for research qualifying for a certain type of review, it always remains within the purview of the local institution under which the IRB is housed to implement more stringent policies and practices related to the protection of human subjects involved in research based on local context and other factors.

Generally speaking, exempt research is often reviewed using a less formal, yet still thorough process, where the determination of exemption can be made independently by a designated member of the IRB or an IRB Administrator who is delegated responsibility for making such determinations. The federal regulations from the Office of Human Research Protections do not require that exempt determinations be made by IRB professionals; however, it is considered a best practice to consult with an IRB professional regarding whether the research meets criteria for exemption. The application for exemption should therefore cover many of the same relevant areas as a non-exempt IRB application and protocol that undergoes expedited or full board review. In order for the determination of exemption to be made, sufficient and explicit information must be provided regarding the purpose and scope of the study, the voluntary nature of participation, recruitment of participants, informed consent process, and data analysis and storage in the same level of detail as a non-exempt protocol. The difference is that when a determination of exemption is made, it means that the study is "exempt" from IRB review and continuing review and monitoring of the research by the IRB. [Note: research involving prisoners or an incarcerated population never qualifies for exemption]. Even though the research may be classified as "exempt" there is still a presumption that an investigator has a duty to adhere to the ethical principles outlined in the Belmont Report.

There are six categories whereby research may be determined to meet exemption criteria. We describe five of these categories below; the sixth rarely applies to SoTL research because it involves food quality and consumer acceptance studies. 
Table 2

Categories of Exempt Research with Examples

\begin{tabular}{l}
\hline Category for Exemption \\
\hline Category 1 is "research conducted in established or \\
commonly accepted educational settings, involving \\
normal educational practices." For the IRB, "normal \\
educational practices" include two areas: "research on \\
regular and special education instructional strategies, or \\
research on the effectiveness among instructional \\
techniques, curricula, or classroom management \\
methods."
\end{tabular}

Category 2 is "research involving the use of educational tests (cognitive, diagnostic, aptitude, achievement), survey procedures, interview procedures or observation of public behavior." There are two exceptions to this category that make a project not exempt: "if (i) information obtained is recorded in such a manner that human subjects can be identified, directly or through identifiers linked to the subjects; and (ii) any disclosure of the human subjects' responses outside the research could reasonably place the subjects at risk of criminal or civil liability or be damaging to the subjects' financial standing, employability, or reputation." An exception to this category is research that involves surveying children or observing children's behavior if the investigator is involved in the observation.

Category 3 is "research involving the use of educational tests (cognitive, diagnostic, aptitude, achievement), survey procedures, interview procedures, or observation of public behavior that is not exempt under Category 2, if: (i) the human subjects are elected or appointed public officials or candidates for public office; or (ii) federal statute(s) require(s) without exception that the confidentiality of the personally identifiable information will be maintained throughout the research and thereafter."

Category 4 is "research involving the collection or study of existing data, documents, records, pathological specimens, or diagnostic specimens, if these sources are publicly available or if the information is recorded by the investigator in such a manner that subjects cannot be identified, directly or through identifiers linked to the subjects."

\section{Example of Research Project} Example: An instructor of legal analysis wants to compare his use of the Socratic method with small-group work in two sections of the same class. This kind of classroom research can be categorized as exempt under Category 1 because the instructor is studying the effectiveness of instructional techniques.

Example: A math instructor wants to collect information on whether a new test instrument is impacting her students' learning. This test instrument is a regular component of the class that all students engage with as part of the class activities. This kind of classroom research can be categorized as exempt under Category 2 because the math instructor is studying a particular educational test and how it affects student learning.

Example: A faculty member would like to survey state governors who are taking part in a continuing education program at her institution regarding their experience with the program and applications to their professions. This kind of classroom research can be categorized as exempt under Category 3 because the research subjects are elected officials. Example: A physics instructor is now offering a "blended" version of an introductory course that is incorporating additional technology components. To study the effectiveness of the course, he wants to compare student test responses in 


\begin{tabular}{|l|l|}
\hline & $\begin{array}{l}\text { the blended version with anonymous } \\
\text { test data from an earlier "traditional" } \\
\text { offering of the course. This kind of } \\
\text { classroom research can be } \\
\text { categorized as exempt under } \\
\text { Category 4 because the instructor is } \\
\text { using data from a previous course in a } \\
\text { way that subjects cannot be } \\
\text { identified. }\end{array}$ \\
\hline $\begin{array}{l}\text { Category 5 is "research and demonstration projects } \\
\text { which are conducted by or subject to the approval of } \\
\text { department or agency heads, and which are designed to } \\
\text { study, evaluate, or otherwise examine:(i) Public benefit } \\
\text { or service programs; (ii) procedures for obtaining } \\
\text { benefits or services under those programs; (iii) possible } \\
\text { changes in or alternatives to those programs or } \\
\text { procedures; or (iv) possible changes in methods or levels } \\
\text { of payment for benefits or services under those } \\
\text { programs." }\end{array}$ & $\begin{array}{l}\text { Example: A faculty member at an } \\
\text { institution of higher education has } \\
\text { been asked by the Department of } \\
\text { Education to submit a report on the } \\
\text { effectiveness of her institution's } \\
\text { McNair Scholar program, which is } \\
\text { federally funded. Part of the data } \\
\text { being collected is student's classroom } \\
\text { research projects. This kind of } \\
\text { classroom research can be } \\
\text { categorized as exempt under } \\
\text { Category } 5 \text { because the research is } \\
\text { directed by the DOE to determine } \\
\text { public benefit. }\end{array}$ \\
\hline
\end{tabular}

Definitions above are taken from Protection of Human Subjects, 2009, §45 CFR 46.101.

The distinction between research that is classified as exempt or non-exempt can be confusing in terms of interpreting and applying the criteria for a certain type of review. Even when research may appear to qualify for exemption, the research design, methodology or instruments may impose radically different procedures or present specific situational risks that in some way deviate from those that a research subject might normally encounter in everyday life. Implicit in research categorized as exempt is the notion that there is little, if any, associated risk. Even when the criteria for exemption appears relevant, if a determination is made that the threshold for the level of risk normally associated with exempt research is surpassed, then the research may be referred for expedited or full board review.

The Department of Health and Human Services recognizes that not all human subjects research warrants review by the full IRB, hence categories of research were developed for nonexempt human subjects research that may undergo an expedited review procedure. The term "expedited" is sometimes perceived as a less time consuming and perhaps less scrutinizing process. This misnomer can create confusion and a source of frustration among faculty members because expedited reviews, depending on the research study, can take time to approve (this is particularly true if revisions are suggested or more information is needed from the researcher before a determination can be made about whether to approve the protocol). When a research project meets criteria for expedited review, this means that the research is not exempt, but it also does not meet the threshold needed to undergo review by a convened meeting of the full IRB. In particular, expedited research must be categorized as minimal risk to subjects in order to undergo expedited review. While regulations provide minimum criteria for research to qualify for 
expedited review (for example that it must not present more than minimal risk to subjects), it is local IRB policy that dictates the review process, and this process often varies based on institution. The IRB may determine a research project's eligibility for expedited review based on federal guidelines, local institutional policies, and requirements of any sponsoring agencies.

Typically, expedited review is conducted using a one or two IRB-member system of review. The two-member system is often referred to as a primary and secondary reviewer system and the review process involves close communication and coordination between the reviewers and compliance staff to reach consensus on the approval of the research. Although the review is normally conducted by one or two IRB members, all IRB members must be provided access to the study and may weigh in on the review and approval process. During expedited review there may be feedback provided to the investigator from reviewers that is relayed through the IRB professional staff to which an investigator must respond before gaining IRB approval.

Example: The same math instructor that was exempt under Category Two above wants to collect information on her students' learning with a particular test instrument, but she plans to create a separate survey that is not a part of normal course assessment to gather additional data. The students will be informed about the anonymous online survey and will be able to choose whether or not to complete the survey on their own time. Although the survey could be offered after grades have been submitted to avoid any perception of coercion, the instructor would prefer to offer the survey immediately after the test instrument has been used, which is in the middle of the semester. Despite the survey being anonymous, based on the demographics of the class and the questions being asked, there is a potential for answers being identifiable and thus a potential risk for coercion if the students are asked to complete the survey during the semester before they have received their final grades. To make sure that students are not experiencing unnecessary risk, this kind of classroom research would go through expedited review.

Full board review is applied to classroom research protocols when they exceed the threshold of minimal risk or deal with a vulnerable population that may warrant more oversight and monitoring by the IRB. Additionally, research that involves deception or that asks for a waiver of informed consent or a waiver of documentation of consent may require full board review. The regulations do not explicitly define what research must be reviewed by the full IRB, but instead outlines criteria for determining what types of research might qualify for exemption as well as those that meet criteria for expedited review. The criteria established by the federal government for both exemption and expedited review represent minimum criteria; therefore, IRBs have much latitude and responsibility for determining the thresholds for research that must undergo review by the full Board. The Common Rule stipulates that expedited review procedures may be used "for certain kinds of research involving no more than minimal risk, and for minor changes to approved research" (Protection of Human Subjects, 2009, § 46.110) Thus, when research presents more than minimal risk to subjects, review by the full IRB is required. The Common Rule defines minimal risk as "the probability or magnitude of harm or discomfort anticipated in the research are not greater in and of themselves than those ordinarily encountered in daily life or during the performance of routine physical or psychological examination or tests" (Protection of Human Subjects, 2009, § 46.102). An increase in risk to participants in areas of social and behavioral research may be perceived and occur in many ways; for example, by recruiting vulnerable populations as research participants; audio or video-taping participants; using deception; and/or collecting information on participants that if confidentiality is breached, could significantly harm an individual's reputation, cause social stigmatization, or result in criminal liability. While these "triggers" for determining full board review are somewhat 
subjective, they often are covered in institutional policies that also serve to define standard practices in the field.

Instances in which SoTL research would need to undergo review by the full IRB are rare. However, there are situations that might very well precipitate full review. For example, a number of federal funding initiatives allow prisoners access to post-secondary education and some colleges and universities have collaborations with prison systems to provide prisoners this access to higher education. Research involving prisoners or other incarcerated individuals is one of the exceptions to exemption; although no similar regulatory exception exists with respect to expedited review, standard practice in the field is that prisoner research, however innocuous, normally requires full review. This institutional policy then might require that an anonymous survey designed for the purpose of SoTL research and administered to a segment of a prison population who is participating in a post-secondary education program undergo review by the full IRB. Research methods that an IRB might consider as warranting full IRB review might include audio or videotaping focus group sessions or deceiving participants as to the purpose of the research. Deception might be a necessary condition to pursue the purpose and aim of the research, however, its use might necessitate full board review.

Example: If the same math teacher used in the preceding examples was gathering the same information with a group of students that could be classified as "at-risk" (for example, they are on academic probation and are taking the math course after already failing it once before), then the IRB may want to explore if this group could be put at additional risk if they were to participate in the study. In the case of "at-risk" students, the IRB may be concerned that the students' reputation might suffer if included in a study, particularly if the group is small enough that participants might be identifiable when reporting the study's findings. Additionally, if this instructor planned to use class time to conduct additional assessments that were outside of normal course procedures, a full review might be conducted to explore whether the use of class time for research would put the students at further academic risk.

\section{Conclusion}

Classroom research is a crucial component to growing our knowledge about how students learn and to better understand effective classroom practice. The information above can provide a clearer pathway for instructors who may be new to classroom research or for more experienced researchers who have yet to conduct research that involves human subjects. To review, we offer the following steps as a guideline for faculty considering SoTL research:

1) Develop a relationship with the IRB professional(s) at your institution so that you know who to contact for questions about the IRB application, protocol, and review process;

2) With the help of the IRB professional(s) at your institution, determine if your classroom investigation constitutes research of human subjects according to the federal regulations used by the IRB;

3) Based on several factors (including risk to participants and vulnerability of study population, among others), and with the help of an IRB professional at your institution, determine the type of review appropriate for your study.

It is important to note that each institution may handle their IRB review with slightly different practices than those described above, but the majority of the above recommendations are based on federal regulations and best practices in the field. SoTL researchers should always ask their institution's IRB professional if they are unsure which type of review is appropriate for 
a classroom research project, or if they unsure of how to proceed with an IRB application or during a review process.

\section{References}

45 C.F.R. § 46 (2009). Retrieved November 5, 2013 from

http://www.hhs.gov/ohrp/humansubjects/guidance/45cfr46.html

Booth, W.C., Colomb, G.G. \& Williams, J.M. (2008). The Craft of Research. Chicago:

University of Chicago Press. doi: 10.7208/chicago/9780226062648.001.0001

Boyer, E.L. (1990). Scholarship Reconsidered: Priorities of the Professoriate. Stanford, CA: The Carnegie Foundation for the Advancement of Teaching.

Brew, A. (2001a). The Nature of Research: Inquiry in Academic Contexts. New York: Routledge.

Brew, A. (2001b). Conceptions of Research: a Phenomenographic Study. Studies in Higher Education, 26 (3), 271-285. doi: 10.1080/03075070120076255

Burman, M.E. \& Kleinsasser, A. (2004). Ethical Guidelines for Use of Student Work: Moving from Teaching's Invisibility to Inquiry's Visibility in the Scholarship of Teaching and Learning. The Journal of General Education, 53 (1), 59-79. doi: 10.1353/jge.2004.0018

Cambridge, B. (1999). The Scholarship of Teaching and Learning: Questions and Answers from the Field. AAHE Bulletin, 52 (4), 7-10.

Hammack, F.M. (1997). Ethical Issues in Teacher Research. Teachers College Record, 99 (2), 247-265.

Huber, M.T. \& Hutchings, P. (2005). The Advancement of Learning: Building the Teaching Commons. San Francisco, CA: Jossey-Bass.

Humphreys, L. (1970). Tearoom Trade: Impersonal Sex in Public Places. London: Duckworth.

Hutchings, P. (2003). Competing Goods: Ethical Issues in the Scholarship of Teaching and Learning. Change: The Magazine of Higher Learning, 35 (5), 26-33. doi:

10.1080/00091380309604116

Kreber, C. (2001). Conceptualizing the Scholarship of Teaching and Identifying Unresolved Issues: The Framework for this Volume. In C. Kreber (Ed.), New Directions for Teaching and Learning: No. 86. Scholarship revisited: Perspectives on the Scholarship of Teaching and Learning (pp. 1-19). San Francisco, CA: Jossey-Bass. 
Lopus, J.S., P.W. Grimes, W.E. Becker \& R.A. Pearson. (2007). Effects of Human Subjects Requirements on Classroom Research: Multidisciplinary Evidence. Journal of Empirical Research on Human Research Ethics: An International Journal, 2 (3), 69-78.

McKinney, K. (2007). Enhancing Learning through the Scholarship of Teaching and Learning: The Challenges and Joys of Juggling. San Francisco: Anker Publishing.

Menges, R.J., Weimer, M., and Associates. (1996). Teaching on Solid Ground: Using Scholarship to Improve Practice. San Francisco, CA: Jossey-Bass.

Milgram, S. (1963). Behavioral Study of Obedience. Journal of Abnormal and Social Psychology, 67, 371-378. doi: 10.1037/h0040525

Pecorino, P.A., Kincaid, S. \& Gironda, B. (2008). Research and Experimentation in Teaching Effectiveness: The Ethical Review Process and the IRB. International Journal for the Scholarship of Teaching and Learning, 2 (1), 1-11.

Pritchard, I.A. (2002). Travelers and Trolls: Practitioner Research and Institutional Review Boards. Educational Researcher, 31 (3), 3-13. doi: 10.3102/0013189X031003003

Pritchard, I.A. (2001). Searching for 'Research Involving Human Subjects:' What is Examined? What is Exempt? What is Exasperating? IRB: Ethics and Human Research, 23 (3), 5-13. doi: $10.2307 / 3564509$

Richlin, L. (2001). Scholarly Teaching and the Scholarship of Teaching. In C. Kreber (Ed.), New Directions for Teaching and Learning: No. 86. Scholarship revisited: Perspectives on the Scholarship of Teaching and Learning (pp. 57-68). San Francisco, CA: Jossey-Bass.

Savory, P., Burnett, A.N. \& Goodburn, A. (2007). Inquiry into the College Classroom: A Journey Toward Scholarly Teaching. Bolton, MA: Anker Publishing.

Schuman, S. H., Olansky, S., Rivers, E., Smith, C.A., Rambo, D. S. (1955). Untreated Syphilis in the Male Negro; Background and Current Status of Patients in the Tuskegee Study. J Chronic Dis, 2 (5), 543-558. doi: 10.1016/0021-9681(55)90153-3

Shulman, L.S. (1999). Taking Learning Seriously. Change: The Magazine of Higher Learning, 31 (4), 10-17. doi: 10.1080/00091389909602695

Stenhouse, L. (1981). What Counts as Research? British Journal of Educational Studies, 29 (2), 103-114. doi: 10.1080/00071005.1981.9973589

Swenson, E.V. \& McCarthy, M.A. (2012). Ethically Conducting the Scholarship of Teaching and Learning Research. In R.E. Landrum \& M.A. McCarthy (Eds.), Teaching Ethically: Challenges and Ppportunities (pp.21-29). Washington, D.C.: American Psychological Association. doi: 10.1037/13496-002 
Linder, K.E., Elek, E.D., \& Calderon, L.

Wright, M.C., C.J. Finelli, D. Meizlish \& I. Bergom. (2011). Facilitating the Scholarship of Teaching and Learning at a Research University. Change: The Magazine of Higher Learning, 43 (2), 50-56. doi: 10.1080/00091383.2011.550255 